\title{
Astrometry of Supernovae in Recent Years
}

\author{
Hitoshi Yamaoka \\ Department of Physics, Faculty of Science, Kyushu University, 4-2-1, \\ Ropponmatsu, Chuo-ku, Fukuoka 810-8560, Japan
}

\begin{abstract}
The positions (the coordinates and the offset from the nucleus of the host) of extragalactic supernovae (SNe) are very important for further research into their nature. The positions reported in the literature are, however, sometimes erroneous. Here we give the precise coordinates of three SNe based on the recently-developed star catalogs. The standard deviations of the positions are expected to be sub-arcsecond, which can be better than the offsets from the ambiguous nuclei of the host galaxies.
\end{abstract}

\section{Introduction}

Astrometric information on extragalactic $\mathrm{SNe}$ is quite important for research into their nature. For example, it has been proposed that there are relationships between the luminosities of SNe Ia and their distances from the nuclei of the host galaxies (e.g. Wang et al, 1997), thus the chemical compositions of the progenitors. Precise positions are also desired for follow-up and future observations of their remnants.

Early reports on the coordinates or offsets provided for the discoveries are, however, sometimes erroneous, as are the cataloged positions of such SNe because there is little additional information about the positions. Such SNe will cause confusion in the future. Here we will give some extreme cases and their precise coordinates based on recently-developed star catalogs (GSC or USNOA2.0).

\section{The extreme cases}

\subsection{SN $1998 \mathrm{~T}$ in NGC 3690}

In this case, the coordinates reported by the discoverer were only accurate to $1 \mathrm{~s}$ and 0'.1. The host galaxy has no apparent nucleus, so the relative offsets from the nucleus cannot be derived. Furthermore, the host galaxy is the eastern part of a complex of galaxies, and many catalogs have identified it as IC 694 but others as NGC 3690. The blobby nature of the host galaxy has led follow-up observers to the misidentification of the SN.

We have examined the literature and found that the interacting pair of galaxies should be called NGC 3690. Using the image taken by M. Yamamoto (Ayabe Astronomical Observatory), we have derived the precise position of SN 1998T with GSC 1.1 reference stars (Yamaoka et al., 1998). 


\subsection{SN 1999et in NGC 1643}

For this SN, the originally reported coordinates were also only accurate to $0.1 \mathrm{~s}$ and 1", but the coordinates and the offsets were inconsistentat a level several times these values. M. Yamamoto imaged this galaxy soon after recognizing the problem, including the new object and another star overexposed on the bulge of the galaxy. We monitored the two objects over 1 month, and determined which was the SN by its declining luminosity. We have derived the position with GSC 1.2 stars (Yamaoka and Yamamoto, 2000).

\subsection{SN $2000 \mathrm{~cm}$ in the anonymous galaxy}

Also for this $\mathrm{SN}$, the original coordinates were nominally accurate to $0.1 \mathrm{~s}$ and 1", but as with SN 1999et the offsets were inconsistent with the coordinates. I have measured the position on the image taken by F.M. Ewalt. Jha et al., 2000 also reported precise coordinates which are in good agreement with our results.

\section{Conclusion}

The precise coordinates for these extreme cases are given in Table 1. The last column gives the catalog names of the reference stars used for the astrometry.

\begin{tabular}{l|rrr|c}
\hline SN name & \multicolumn{3}{|c|}{ R.A. (2000) Decl. } & reference \\
\hline SN 1998T & $11^{\mathrm{h}} 28^{\mathrm{m}} 33^{\mathrm{s}} .16$ & $+58^{\circ} 33^{\prime}$ & $43^{\prime \prime} \cdot 7$ & GSC 1.1 \\
SN 1999et & $4^{\mathrm{h}} 43^{\mathrm{m}} 43^{\mathrm{s}} .53$ & $-5^{\circ} 19^{\prime}$ & $19^{\prime \prime} .8$ & GSC 1.2 \\
SN 2000cm & $12^{\mathrm{h}} 12^{\mathrm{m}} 59^{\mathrm{s}} .43$ & $+7^{\circ} 18^{\prime}$ & $00^{\prime} .8$ & USNO A2.0 \\
\hline
\end{tabular}

Table 1. The precise coordinates of SNe.

The standard deviations of SNe positions are of sub-arcsecond order. The reference star catalogs themselves and the accessibility of them have been greatly improved the last three years. A standard CCD image has sufficient reference stars for the astrometry.

The offsets from the ambiguous nuclei of the host galaxies give less accurate positions than the coordinates derived with appropriate reference stars, though they are useful for cross checking. The positions of the galaxies given in the catalog are also sometimes erroneous.

Acknowledgments. The star catalogs were retrieved from the NAOJ ADC server.

\section{References}

Jha, S., Challis, P., Kirshner, R., \& Yamaoka, H. 2000, IAU Circular No. 7438 Wang, L., Hoflich, P., \& Wheeler, J. C. 1997, ApJ, 483, L29

Yamaoka, H., Kato, T., Filippenko, A. V., \& Van Dyk, S. D. 1998, IAU Circular No. 6859

Yamaoka, H., \& Yamamoto, M. 2000, IAU Circular No. 7344 